\title{
Update on the management of prostate cancer with goserelin acetate: patient perspectives
}

This article was published in the following Dove Press journal:

Cancer Management and Research

12 August 2009

Number of times this article has been viewed

\section{Shandra Wilson \\ Division of Urology, University of Colorado, Aurora, CO, USA}

Correspondence: Shandra Wilson Associate Professor, Director Uro Oncology Fellowship, University of Colorado, I263I E. 17th Ave., MS 319, Aurora, CO 80045, USA Email shandra.wilson@uchsc.edu

\begin{abstract}
The guidelines for the use of androgen deprivation therapy (ADT) have changed significantly over the last 5 years. This paper reviews the current recommendations and documents the reasons for these changes, in a review of the world's literature on ADT over the last 5 years. Special emphasis on randomized controlled trials and high-impact journals was included in the Medline search and review. One hundred articles on this topic written in the last 5 years were reviewed. Fifty-nine contained nonindustry-biased findings in major-impact journals and were available in English. The benefits of ADT are evident in several areas, including neoadjuvantly and adjuvantly in patients treated with external beam radiation therapy for intermediate- and high-risk disease; in patients who have undergone prostatectomy and who are found to have lymph node involvement on surgical resection; in high-risk patients after definitive therapy; and in patients who have developed symptomatic local progression or metastasis. This paper reviews the risks and benefits in each of these scenarios and the risks of androgen deprivation in general, and delineates the areas where ADT was previously recommended, but has been found to no longer be of benefit.
\end{abstract}

Keywords: prostate cancer, goserelin acetate

\section{Definition}

Androgen deprivation is defined as a lowering of serum testosterone through the administration of an LHRH (leuteinizing hormone releasing hormone) agonist (unless otherwise specified) throughout this paper.

\section{Introduction}

The use of androgen deprivation therapy (ADT) for prostate cancer increased in the early part of the 21 st century. In fact, its use increased so significantly, the payments for ADT constituted the second highest expense for Medicare part B expenditure in $2003 .{ }^{1}$ The realization of this magnitude led to careful scrutiny of the risks and benefits of therapy as well as the restructuring of reimbursement for ADT by Medicare. This paper reviews the findings of investigations into the risks and benefits of ADT, delineates areas where its use has been deemed inappropriate/ineffective, and summarizes the current clinical situations where the use of ADT remains recommended.

The risks of ADT are becoming more fully elucidated. Multiple retrospective and prospective studies have been published recently, defining these risks. The administration of ADT can cost a patient physically, financially, and emotionally.

\section{Adverse events associated with androgen deprivation} It is now well understood that the decreased lean body mass and increased body fat composition seen grossly with the administration of $\mathrm{ADT}^{2}$ is correlated with negative 
changes in the serum lipid profile, an increased risk of insulin resistance and an increased risk of coronary artery disease. This increase in cardiovascular disease correlates with an increase in myocardial infarction and even sudden cardiac death in some studies. ${ }^{3}$ This risk of the development of diabetes and coronary artery disease has been confirmed in other studies and appears to be even greater in men over 65 years of age. ${ }^{4}$ The description of this "metabolic syndrome" and the concern about its impact on survival on patients treated with ADT led to the reanalysis of the RTOG 92-02 trial. In this trial 1554 men with locally advanced prostate cancer were treated with neoadjuvant goserelin for 4 months prior to radiation therapy and either no additional therapy or for 24 additional months. Although a striking increase in death from coronary artery disease was not seen in the men who underwent prolonged treatment with ADT, the authors did note that "although there was a significant advantage for all prostate cancer-specific end points [with prolonged therapy], the longer-term arm of ADT in RTOG 92-02 was associated with greater noncancer mortality than [the] short-term [arm]." They go on to state, "Compared to the general population, men with prostate cancer [are known to] have higher rates of non-cancer death and GnRH agonists may contribute to this through multiple mechanisms". ${ }^{5}$ An interim analysis of a recent prospective randomized trial showed a reversal of the negative effects on low density lipoprotein (LDL), very low density lipoprotein (VLDL), and high density lipoprotein (HDL) with the administration of toremofine, ${ }^{6}$ a similar effect would likely be seen with 3-hydroxy-3-methylglutarylcoenzyme A reductase inhibitors, or "statins." A randomized prospective trial from Scandinavia showed that the use of estrogen, compared to ADT, decreased the risk of this "metabolic syndrome" as well as the risk osteoporosis but not surprisingly also increased the risk for thromboembolic event for a net negative improvement in adverse events with therapy. ${ }^{7}$ It is recommended at this time to screen carefully and treat all patients on ADT for hyperlipidemia, diabetes, and coronary artery disease. ${ }^{8}$
The risk of osteoporosis with ADT is real, appears early, and increases the risk of fracture. ${ }^{9}$ Unfortunately, hip fracture is a powerful independent predictor of early mortality. ${ }^{10}$ It is recommended that all men over 50, and particularly those treated with ADT be supplemented with 800 to $1000 \mathrm{IU}$ of vitamin D, $1200 \mathrm{mg}$ of calcium daily, frequent weight-bearing exercise, and be screened for osteoporosis regularly. ${ }^{11}$ Those at higher risk for fracture should be treated with bisphosphonates. A prospective randomized study of alendronate (70 mg weekly by mouth vs placebo) started at the initiation of ADT showed improvement of spine and hip densities and this finding was less significant if it was initiated after just one year of ADT. ${ }^{12}$ Quarterly or annual $4 \mathrm{mg}$ zolendronic acid infusions for men with normal creatinine shows similar or even greater benefits, although the rare risk of osteonecrosis can be devastating. ${ }^{12-14}$ It is the official recommendation of the National Comprehensive Cancer Network that zoledronic acid be administered to men with bony progressive metastatic prostate cancer on ADT to help prevent skeletal related events.

Although it would seem the financial costs of ADT are minimal, a recent study showed, if used for a significant amount of time, the cost of ADT would quickly be greater than that of radical prostatectomy or external beam therapy. ${ }^{15}$ At a time when the cost to the government to cover medical costs for its citizens is rapidly approaching one half of the United States budget, this is not a trivial issue.

In addition to the adverse events seen with ADT on the cardiovascular, skeletal, and financial system, ADT may also have an effect on cognition and physical function. A retrospective trial showed that up to $27 \%$ of patients on ADT suffered a diagnosable psychiatric illness during their treatment, and that in patients on ADT tested over time, many lost cognition in one, if not two, measurable areas. ${ }^{16,17}$ A recent prospective controlled study of men on androgen deprivation more than 6 months found a decline of lower body physical function with statistically slower walk and chair-rise times with treatment. ${ }^{18}$ A retrospective study from the Mayo Clinic in Scottsdale found that the average hemoglobin

Table I Summary of androgen deprivation indications and recent changes

\begin{tabular}{|c|c|}
\hline Less evidence for ADT in 2009 & Still good uses for ADT in 2009 \\
\hline Localized dz - prior to prostatectomy ${ }^{21}$ & Local/advanced dz - PSA $>50$, PSADT $<12 \mathrm{mo}^{29}$ \\
\hline Localized dz - prior to EBXRT, brachy $\mathrm{tx}^{24-27}$ & Local/advanced dz - prior to EBXRT ${ }^{43-47}$ \\
\hline Localized dz - no primary treatment ${ }^{28}$ & Local obstructive $s x / m e t a s t a t i c ~ d i s e a s e^{52}$ \\
\hline \multirow[t]{2}{*}{ Biochemical recurrence after tx, slow PSADT ${ }^{34}$} & Biochem recurrence $p$ tx, high-risk Cap death ${ }^{36-38}$ \\
\hline & Lymph node involvement at prostatectomy ${ }^{49}$ \\
\hline
\end{tabular}

Abbreviations: ADT, androgen deprivation therapy; EBXRT, external beam radiation; PSADT, prostate-specific antigen doubling time; dz, disease; tx, treatment; sx, symptoms; p, after; Cap, prostate cancer. 
drop on patients treated with LHRH antagonists is $1.6 \mathrm{~g} / \mathrm{dL}$ which may contribute to the physical decline seen in these patients. ${ }^{19}$ As previous studies have found a profound and prolonged suppression of testosterone long after the cessation of LHRH analogues ( $53 \%$ of men remaining castrate up to 2.5 years who had been on ADT for 4 or more years), ${ }^{20}$ there is concern that the adverse risks of coronary artery disease, diabetes, osteoporosis, cognitive/physical changes, and anemia could persist beyond active therapy as well.

Although the risks of ADT are justified in some situations, the increased awareness of their magnitude, as well as data pointing to questionable efficacy have caused a shift in the recommendation to withdraw the recommend ADT in several clinical scenarios which will be reviewed below.

\section{Androgen therapy not recommended}

First, support for neoadjuvant androgen deprivation prior to or after prostatectomy has waned in men with localized disease. Although many studies have been done evaluating its efficacy and a possible decrease in positive surgical margins has been seen, no improvement in overall survival has been documented, making justification of the risks of ADT null. ${ }^{21}$ One of the largest prospective randomized prostate cancer trials, the Early Prostate Cancer (EPC) trial, evaluated $150 \mathrm{mg}$ of bicaludamide daily in addition to standard therapy for men with low- and high-risk disease. Analysis of the overall trial did not show any advantage for its use in low-risk patients treated with surgery, radiation, or observation. Although in general antiandrogen monotherapy is thought to be inferior and less well tolerated that traditional androgen deprivation, ${ }^{22}$ the study did suggest some benefit in higher-risk patients with locally advanced or micro-metastatic disease in progression-free survival, but there was no benefit in overall survival. ${ }^{23}$

Similarly, although earlier studies might have suggested some benefit for neoadjuvant androgen deprivation prior to external beam radiation for low-risk patients, with higher dosing, ${ }^{24}$ conformal techniques that appear to make doses of 70 to 79 Gy possible with minimal toxicity, ${ }^{25}$ and the wider availability of brachytherapy, translate that, in general, neoadjuvant androgen deprivation is no longer recommended prior to radiation therapy for patients with low-risk prostate cancer. ${ }^{26,27}$

In fact, the results of a large retrospective trial recently published in JAMA evaluated 19,271 with localized prostate cancer. This study found that there was no increase in 10-year overall survival in men treated with androgen deprivation compared to conservative management, and indeed, there was a lower 10-year prostate-cancer specific survival in men treated with primary androgen deprivation. ${ }^{28}$ Similarly, when Dr Studer reported on the results of the EORTC trial where in 939 men with prostate cancer not suitable for local curative treatment were evaluated after their randomization to immediate vs deferred ADT, he concluded that "Patients with a baseline PSA $>50 \mathrm{ng} / \mathrm{mL}$ and/or a PSADT [prostate-specific antigen doubling time] $<12$ months were at increased risk to die from prostate cancer and might have benefited from immediate ADT, whereas patients with a baseline PSA $<50 \mathrm{ng} / \mathrm{mL}$ and a slow PSADT ( $>12$ months) were likely to die of cuases unrelated to prostate cancer, and thus could be spared the burden of immediate ADT". ${ }^{29}$ The group of men with low risk tumors who do not get treated with ADT may benefit from active surveillance where similar rates of efficacy are achieved with decreased morbidity compared to definitive therapy or androgen deprivation. ${ }^{30,31}$ With large trials suggesting little benefit seen in screening for prostate cancer (or at least a large number needed to treat to see benefit), particularly for men over 70, the cohort who may have previously been treated with ADT due to inability to tolerate definitive therapy may now be likely to avoid screening, diagnosis and overtreatment with androgen deprivation as well. ${ }^{32,33}$

Data following the natural course of men with recurrence after primary therapy show that very few die of their disease and that frequently competing causes of death and death from prostate cancer-specific death are equally likely upon recurrence of disease. ${ }^{34} \mathrm{~A}$ literature review published in $J A M A$ concluded that "although patients with increasing prostate-specific antigen levels after local treatment without metastatic disease frequently undergo ADT, the benefits of this strategy are not clear... and need to be weighed carefully against substantial risks and adverse effects on quality of life". ${ }^{35}$ This observation has led to the recommendation of a risk-stratified approach. This approach includes the incorporation of known risk factors for recurrent aggressive disease to define which patients need more aggressive, early therapy and which patients may be able to be spared the adverse events of androgen deprivation at the detection of biochemical recurrence. The elements of risk-stratification may include pretreatment PSA, PSA velocity, Gleason score, volume of tumor or stage,${ }^{36-38}$ PSA velocity or total value, ${ }^{29,39}$ PSA nadir and time to recurrence after therapy ${ }^{40,41}$ and possibly the presence of circulating tumor cells, ${ }^{42}$ as all of these have been shown to be associated with increased risk of progression or death from prostate cancer. 


\section{Androgen therapy recommended}

Clearly the risk of ADT is worth the possible benefit in men with high stage, high grade tumors, with rapid recurrence after therapy, doubling times under 12 months and/or total PSA values greater than $50 \mathrm{ng} / \mathrm{mL}$. These men should also have consultation with medical oncology early and should be considered strongly for clinical trials.

Additional scenarios where ADT should be strongly considered and has been proven to provide benefit include: neoadjuvantly/adjuvantly ADT in patients with intermediate/ high-risk prostate cancer treated with external beam radiation therapy; adjuvantly after prostatectomy in patients found to have lymph node involvement on surgical resection; and in patients who have developed (symptomatic) bony metastasis or locally obstructive symptoms. Support for ADT in these situations will be described below.

The administration of neoadjuvant ADT remains an independent predictor of long-term control in patients with intermediate and high risk cancer treated with external beam radiation (EBXRT) and should be given prior to therapy. ${ }^{43,44} \mathrm{~A}$ randomized trial of 802 Australian men found that 3 months of neo-adjuvant ADT showed an inferior prostate cancer-specific mortality to men treated with 6 months in men with locally advanced prostate cancer, so a treatment with 4 to 6 months of neoadjuvant ADT is recommended prior to external beam radiation. ${ }^{45}$ The initial finding in the randomized controlled RTOG/ EORTC trials showing that an additional 24 to 36 months of ADT after EBXRT improves survival in men with high risk disease and is recommended as well. ${ }^{46,47}$ In these trials androgen deprivation has been achieved using an LHRH agonist as well as an antiandrogen. Similar efficacy may be able to be achieved with LHRH monotherapy, but formal comparative studies have not been performed. Interestingly, when a group in Spain looked at predictors for hematuria in patients who had undergone conformal prostate radiation for cancer, the administration of adjuvant androgen deprivation was protective (a factor of 5) for the development of hematuria (whereas transurethral resection [TURP] increased the risk three times) ${ }^{48}$

There is support for immediate ADT in men found to have lymph node involvement at prostatectomy. Only one randomized, controlled has been performed to investigate this situation to date. Although this trial is small and may have exaggerated survival advantages by delaying the initiation of ADT in the initially nontreated group, the study showed a significant improvement in overall, disease-specific, and progression-free survival advantage for patients who undergo early ADT in this setting. ${ }^{49}$

In men wishing to undergo brachytherapy, but who have a prostate too large to be treated effectively, ADT can be considered. Similar results may be achieved with 5-alpha reductase inhibitors or even antiandrogens alone as well (with appropriate prevention of gynecomastia), and may be preferred. $^{50}$

In men wishing to undergo salvage or primary cryotherapy for prostate cancer, the largest series in the literature reports success when ADT is used adjuvantly. This strategy may be beneficial with this treatment modality. ${ }^{51}$ Meaningful studies comparing ADT with cryotherapy to cryotherapy alone have not been performed to my knowledge.

Finally, data from the MRC trial published in 1997 showing that ADT helps decrease symptoms from patients with advanced disease is still to be considered today. This prospective randomized trial of 938 men with locally advanced or asymptomatic metastatic prostate cancer showed that rates of pathological fracture, spinal cord compression, urteric obstruction, the development of extra-skeletal metastases (and pain from these metastases) as well as death from prostate cancer are statistically more common in men who were not treated with early androgen deprivation. ${ }^{52}$ It is important to note that since the introduction of PSA screening there has been a significant stage migration downward, so patients with this level of disease burden may be less common.

\section{Future research}

Additional investigation to the risk and benefit of the primary use or adjuvant use of other agents in this area such as somatostatin or NF-kB ligand (RANKL) receptor antagonists, RNF6-ubiquitiniation inhibitors, aromatase inhibitors, or long-term antiandrogen monotherapy ${ }^{23,53-55}$ is being performed. The use of agents to reduce production of dihydrotestosterone in the prevention of prostate cancer is interesting, but so far does not appear to improve the efficacy of complete androgen blockade in men with castration recurrent prostate cancer. ${ }^{56}$ The use of 1alpha, 25-dihydroxy vitamin D3 downregulates the expression of prostate specific membrane antigen in prostate cancer cells. ${ }^{57}$ There is a possibility that these agents may improve the efficacy profile of ADT and/or decrease its side effect profile. LHRH antagonists have not been fully studied in all of the scenarios mentioned above but are predicted to have a similar effect to LHRH agonists given with an antiandrogen for the first seven days as their suppression of 
testosterone is similar. ${ }^{58,59}$ Additionally, it will be exciting to see if new pharmaceutical agents such as abiraterone and MDV 3100, which inhibit testosterone in different ways, have as much promise as is anticipated with additional study.

\section{Summary}

In summary, the risks of ADT have been more elaborated over the last 5 years and are significant. Patients should be appraised of these risks and should undergo monitoring for hyperlipidemia, coronary artery disease, osteoporosis, and diabetes while castrate. The initiation of calcium and vitamin D therapy are recommended with the initiation of ADT (for men who are not already on it), with the consideration of oral of intravenous bisphosphonates for those with metastatic disease or osteoporosis. ADT is discouraged in conjunction with prostatectomy unless local lymph nodes are found to be involved. ADT is generally not recommended with adequate ( 70 to 79 Gy) conformal (145 Gy), or brachytherapy radiation in patients with low-risk disease. And ADT should not be considered primary therapy for men with low-risk prostate cancer. In men with a biochemical (PSA-only) recurrence after primary therapy, the timing of the initiation of ADT should be considered carefully. Those patients with high stage ( $\geq 2 b$ ), high grade tumors $\geq 7$, with rapid PSA velocities before and/or after treatment (total PSA $>10 \mathrm{ng} / \mathrm{mL}$ before treatment, doubling time $<12$ months or absolute increase of $2 \mathrm{ng} / \mathrm{mL} /$ year before or after treatment), PSA nadir $>0.2 \mathrm{ng} / \mathrm{mL}$, or overall high total PSA $(>50 \mathrm{ng} / \mathrm{mL})$ likely benefit from early intervention and possibly from involvement in a clinical trial. Additional situations where ADT is strongly recommended are: in a neoadjuvant and setting for 4 to 6 months in men with intermediate risk cancer undergoing radiation therapy or for men with large prostates and low-risk cancer anticipating brachytherapy; in an adjuvant setting for 2 to 3 years in men with high-risk prostate cancer undergoing radiation therapy; in men found to have positive nodes at prostatectomy; and in men with symptomatic (obstructing or painful) locally advanced or metastatic disease. ADT may be considered adjuvantly or neoadjuvantly in patients being treated with cryotherapy. Studies to identify agents that are equally effective but that have fewer adverse effects are ongoing.

\section{Disclosure}

The author declares no conflicts of interest.

\section{References}

1. Brower V. Watchful waiting beats androgen deprivation therapy in early prostate cancer. J Natl Cancer Inst. 2008;100(21): 1494-1496.

2. Van Londen, Gisjsberta J, Levy M, et al. Body composition changes during androgen deprivation therapy for prostate cancer: a 2-year prospective study. Crit Rev Oncol Hematol. 2008;68(2): $172-177$.

3. Keating NL, O'Malley AJ, Smith MR. Diabetes and cardiovascular disease during androgen deprivation therapy for prostate cancer. J Clin Oncol. 2006;24(27):4448-4456.

4. Tsai HK, D'Amico AV, Sadetsky, et al. Androgen deprivation therapy for localized prostate cancer and the risk of cardiovascular morality. J Natl Cancer Inst. 2007;99(20):1516-1524.

5. Efstathiou JA, Kyounghwa B, Shipley WU, et al. Cardiovascular mortality and duration of androgen deprivation for locally advanced prostate cancer: analysis of RTOG 92-02. Eur Eurol. 2008;54(4): 816-824.

6. Smith M, Malkowicz S, Chu F, et al. Toremifene improves lipid profiles in men receiving androgen-deprivation therapy for prostate cancer: interim analysis of a multicenter phase III study. J Clin Oncol. 2008;26(11):1824-1829.

7. Helund PO, Damber JE, Hagerman I, et al. Parenteral estrogen versus combined androgen deprivation in the treatment of metastatic prostatic cancer: part 2. Final evaluation of the Scandinavian Prostatic Cancer Group (SPCG) Study No. 5. Scand J Urol Nephrol. 2008;42(3):220-229.

8. Polly EK, Chase AL, Schultz LM, et al. Increased risk of metabolic syndrome, diabetes mellitus and cardiovascular disease in men receiving androgen deprivation therapy for prostate cancer. Pharmacotherapy. 2008;28(12):1511-1522.

9. Galvao DA, Spry NA, Taaffe DR, et al. Changes in muscle, fat and bone mass after 36 weeks of maximal androgen blockade for prostate cancer. BJU Int. 2008;102(1):44-47.

10. Piirtola M, Vahlberg T, Lopponen M, et al. Fractures as predictors of excess mortality in the aged - a population-based study with a 12-year follow up. Eur J Epidemiol. 2008;23(11):747-755.

11. Flaig TW and Glode LM. Management of the side effects of androgen deprivation therapy in men with prostate cancer. Exp Opin Pharmacother. 2008;9(16):2829-2841.

12. Greenspan SL, Nelson JB, Trum DL, et al. Skeletal health after continuation, withdrawal, or delay of alendronate in men with prostate cancer undergoing androgen-deprivation therapy. J Clin Oncol. 2008;26(27) 4426-4434.

13. Michaelson MD, Kaufman DS, Lee H, et al. Randomized controlled trial of annual zoledronic acid to prevent gonadotropin-releasing hormone agonist-induced bone loss in men with prostate cancer. J Clin Oncol. 2007;25(9):1038-1042.

14. Smith MR, Estham J, Gleason DM, et al. Randomized controlled trial of zoledronic acid to prevent bone loss in men receiving androgen deprivation therapy for nonmetastatic prostate cancer. J Urol. 2003;169(6):2008-2012.

15. Wilson LS, Tesoro R, Elkin EP, et al. Cumulative cost pattern comparison of prostate cancer treatments. Cancer. 2007;109(3):518-527.

16. Nelson CJ, Lee JS, Gamboa MC, et al. Cognitive effects of hormone therapy in men with prostate cancer: a review. Cancer. 2008;113(5):1097-1106.

17. DiBlasio CJ, Hammett J, Malcolm JB, et al. Prevalence and predictive factors for the development of de novo psychiatric illness in patients receiving androgen deprivation therapy for prostate cacner. Can J Urol. 2008; 15(5):4249-4256.

18. Levy ME, Perera SL, Nelson GJ, et al. Physical function changes in prostate cancer patients on androgen deprivation therapy: a 2-year prospective study. Urology. 2008;71(4):735-739.

19. Curtis KK, Adam TJ, Chen S, et al. Anaemia following initiation of androgen deprivation therapy for metastatic prostate cancer: a retrospective chart review. Aging Male. 2008;11(4):157-161. 
20. Bong GW, Clarke HS, Hancock WC, et al. Serum testosterone recovery after cessation of long-term luteinizing hormone-releasing hormone agonist in patients with prostate cancer. Urology. 2008;71(6): 1177-1180.

21. Heidenreich A, Gunnar A, Bolla M, et al. EAU guidelines on prostate cancer. Eur Urol. 2008;53(1):68-80.

22. Iversen $\mathrm{P}$, Johansson JE, Lodding $\mathrm{P}$, et al. Bicalutamide $150 \mathrm{mg}$ in addition to standard care for patients with early non-metastatic prostate cancer: updated results from the Scandinavian Prostate Cancer Period Group-6 Study after a median follow-up period of 7.1 years. Scand J Urol Nephrol. 2006;40(6):441-452.

23. McLeod DG, Iversen P, See WA, et al. Bicalutamide $150 \mathrm{mg}$ plus standard care vs standard care alone for early prostate cancer. BJU Int. 2006;97(2):247-254.

24. Pilepich MV, Winter K, Lawton, CA, et al. Androgen suppression adjuvant to definitive radiotherapy in carcinoma - long-term results of phase III RTOG 85-31. Int J Radiat Oncol Biol Phys. 2002;53(5): 1097-1105.

25. Jereczek-Fossa BA, Vavassori A, Fodor C, et al. Dose escalation for prostate cancer using the three-dimensional conformal dynamic arc technique: analysis of 542 consecutive patients. Int J Radiat Oncol Biol Phys. 2008;71(3):784-794.

26. Mendenhall WM, Henderson RH, Mendenhall NP. Definitive radiotherapy for prostate cancer. Am J Clin Oncol. 2008;31(5): 496-503.

27. Beasley M, Williams SG, Pickles T. Expanded risk groups help determine which prostate radiotherapy sub-group may benefit from adjuvant androgen deprivation therapy. Radiat Oncol. 2008;3:8.

28. Lu-Yao GL, Albertsen PC, Moore DF, et al. Survival following primary androgen deprivation therapy among men with localized prostate cancer. JAMA. 2008;300(2):173-181.

29. Studer UE, Collette L, Whelan P, et al. Using PSA to guide timing of androgen deprivation in patients with $\mathrm{T} 0-4 \mathrm{N0}-2 \mathrm{M} 0$ prostate cancer not suitable for local curative treatment (EORTC 30891). Eur Urol. 2008;52(5):941-949.

30. Steineck G, Helgesen F, Adolfsson J, et al. Quality of life after radical prostatectomy or watchful waiting. $N$ Engl J Med. 2002;347(11): 790-796.

31. Ercole B, Marietti SR, Fine J, et al. Outcomes following active surveillance of men with localized prostate cancer diagnosed in the prostate specific antigen era. J Urol. 2008;180(4):1336-1339.

32. Andriole GL, Grubb RL, Buys SS, et al. Mortality results from a randomized prostate-cancer screening trial. $N$ Engl J Med. 2009;360(13):1310-1319.

33. Schroder FH, Hugosson J, Roobol MJ, et al. Screening and prostatecancer mortality in a randomized European study. $N$ Engl J Med. 2009;360(13):1320-1328.

34. Simmons MN, Stephenson AJ, Klein EA. Natural history of biochemical recurrence after radical prostatectomy: risk assessment for secondary therapy. Eur Urol. 2007;51(5):1175-84.

35. Sharifi N, Gulley J, Dahut WL. Androgen deprivation therapy for prostate cancer. JAMA. 2005;294(2):238-244.

36. Soto DE, Andrige RR, Pan CC, et al. In patients experiencing biochemical failure after radiotherapy, pretreatment risk group and PSA velocity predict differences in overall survival and biochemical failure-free interval. Int J Radiat Oncol Biol Phys. 2008;71(5): 1295-1301.

37. Palma D, Tyldesley S, Pickles T, et al. Pretreatment prostatespecific antigen velocity is associated with development of distant metastases and prostate cancer mortality in men treated with radiotherapy and androgen-deprivation therapy. Cancer. 2008;112(9): 1941-1948.

38. Williams SG, Buyyounouski M, Pickles TK, et al. Percentage of biopsy cores positive for malignancy and biochemical failure following prostate cancer radiotherapy in 3,264 men: statistical significance without predictive performance. Int J Rad Oncol Bio Phys. 2008;70(4): 1169-1175.
39. D'Amico AV, Renshaw AA, Sussman B, et al. Pretreatment PSA velocity and risk of death from prostate cancer following external beam radiation therapy. JAMA. 2007;294(4):440-447.

40. D'Ambrosio DJ, Ruth K, Horwitz EM, et al. How can men destined for biochemical failure after androgen deprivation and radiotherapy be identified earlier? Int J Radiat Oncol Biol Phys. 2008;70(5): 1487-1491.

41. Rodrigues NA, Chen MH, Catalona WJ, et al. Predictors of mortality after androgen-deprivation therapy in patients with rapidly rising prostate-specific antigen levels after local therapy for prostate cancer. Cancer. 2006;107(3):514-520.

42. Okegawa T, Nutahara K, Higashihara E. Immunomagnetic quantification of circulating tumor cells as a prognostic factor of androgen deprivation responsiveness in patients with hormone naïve metastatic prostate cancer. J Urol. 2008;180(4):1342-1347.

43. D'Amico AV, Loffredo M, Renshaw AA, et al. Six-month androgen suppression plus radiation therapy compared with radiation therapy alone for men with prostate cancer and a rapidly increasing pretreatment prostate-specific antigen level. J Clin Oncol. 2006;24(25):4190-4195.

44. Zelefsky MJ, Yamada Y, Fuks Z, et al. Long-term results of conformal radiotherapy for prostate cancer: impact of dose escalation on biochemical tumor control and distant metastases-free survival outcomes. Int J Radiat Oncol Biol Phys. 2008;71(4):1028-1033.

45. Denham JW, Steigler A, Wilcox C, et al. Time to biochemical failure and prostate-specific antigen doubling time as surrogates for prostate cancer-specific mortality; evidence from the TROG 96.01 randomized controlled trial. Lancet Oncol. 2008;9(11):1058-1068.

46. Hanks GE, Pajak TF, Porter A, et al. Phase III trial of long-term adjuvant androgen deprivation after neoadjuvant hormonal cytoreduction and radiotherapy in locally advanced carcinoma of the prostate: the Radiation Therapy Oncology Group Protocol 92-02. J Clin Oncol. 2003;21(21):3972-3978.

47. Bolla M, Collette L, Blank L, et al. Long-term results with immediate androgen suppression and external irradiation in patients with locally advanced prostate cancer (an EORTC study): a phase III randomized trial. Lancet. 2002(9327);360:103-106.

48. Zapatero A, Garcia-Vicente F, Sevillano D, et al. Is hormone therapy a protective factor for late hematuria after high-dose radiotherapy in prostate cancer? Urology. 2008;72(5):1130-1134.

49. Messing E, Manola J, Yao J, et al. Immediate versus deferred androgen deprivation treatment in patients with node-positive prostate cancer after radical prostatectomy and pelvic lymphadenectomy. Lancet Oncol. 2006;7(6):472-479.

50. Petit JH, Gluck C, Kiger WS, et al. Bicalutamide alone prior to brachytherapy achieves cytoreduction that is similar to luteinizing hormone-releasing hormone analogues with less patient-reported morbidity. Urol Oncol. 2008;26(4):372-377.

51. Ghafar MA, Johnson CE, De La Taille A, et al. Salvage cryotherapy using an argon based system for locally recurrent prostate cancer after radiation therapy: the Columbia experience. J Urol. 2001;166(4): 1333-1337.

52. Immediate versus deferred treatment for advanced prostatic cancer: initial results of the Medical Research Council Trial. The Medical Research Council Prostate Cancer Working Party Investigators Group. Br J Urol. 1997;79(2):235-246.

53. Stangelberger A, Shally AV, and Djavan B. New treatment approaches for prostate cancer based on peptide analogues. Eur Urol. 2008;53(5):890-900.

54. Kexin X, Shimelis H, Linn DE, et al. Regulation of androgen receptor transcriptional activity and specificity by RNF6-induced ubiquitination. Cancer Cell. 2009;15(4):270-282.

55. Xu K, Shimelis H, Linn DE, et al. Regulation of androgen receptor transcriptional activity and specificity by RNF6-induced ubiquitination. Cancer Cell. 2009;15(4):245-247.

56. Shah SK, Trump DL, Sartor O, et al. Phase II study of dutasteride for recurrent prostate cancer during androgen deprivation therapy. J Urol. 2009;181(2):621-626. 
57. Serda RE, Bisoffi M, Thompson TA, et al. 1alpha, 25-Dihydroxyvitamin D3 down-regulates expression of prostate specific membrane antigen in prostate cancer cells. Prostate. 2008;68(7):773-783.

58. Klotz L, Boccon-Gibod L, Shore ND, et al. The efficacy and safety of degarelix: a 12-month, comparative, randomized, open-label, parallel-group phase III study in patients with prostate cancer. $B J U$ Int. 2008;102(11):1531-1538.
59. Van Poppel H, Tombal B, de la Rosette JJ, et al. Degareliz: a novel gonadotropin-releasing hormone $(\mathrm{GnRh})$ receptor blocker - results from a 1-yr, multicentre, randomized, phase 2 dosage-finding study in the treatment of prostate cancer. Eur Urol. 2008;54(4):805-813.

\section{Publish your work in this journal}

Cancer Management and Research is an international, peer-reviewed open access journal focusing on cancer research and the optimal use of preventative and integrated treatment interventions to achieve improved outcomes, enhanced survival and quality of life for the cancer patient The journal welcomes original research, clinical \& epidemiological studies, reviews \& evaluations, guidelines, expert opinion \& commentary, case reports \& extended reports. The manuscript management system is completely online and includes a very quick and fair peerreview system, which is all easy to use. Visit http://www.dovepress.com/ testimonials.php to read real quotes from published authors.

Submit your manuscript here: http://www.dovepress.com/cancer-management-and-research-journal 